\title{
Philosophiques
}

\section{Précis de Wittgenstein et la philosophie austro-allemande}

\section{Kevin Mulligan}

Volume 42, numéro 2, automne 2015

URI : https://id.erudit.org/iderudit/1034745ar

DOI : https://doi.org/10.7202/1034745ar

Aller au sommaire du numéro

Éditeur(s)

Société de philosophie du Québec

ISSN

0316-2923 (imprimé)

1492-1391 (numérique)

Découvrir la revue

Citer ce document

Mulligan, K. (2015). Précis de Wittgenstein et la philosophie austro-allemande. Philosophiques, 42(2), 359-365. https://doi.org/10.7202/1034745ar d'utilisation que vous pouvez consulter en ligne.

https://apropos.erudit.org/fr/usagers/politique-dutilisation/ 


\title{
Disputatio
}

\section{Précis de Wittgenstein et la philosophie austro-allemande}

\author{
KEVIN MULLIGAN \\ Université de Genève \\ kevin.mulligan@unige.ch
}

Philosophiques a toujours accueilli avec une générosité extraordinaire mes propositions au sujet de la philosophie austro-allemande. En I999 est paru un numéro consacré à la "Critique de la raison en Europe centrale» (Philosophiques, 26, 2), qui présente plusieurs articles issus d'un colloque que j'ai organisé à Cerisy avec Jean-Pierre Cometti. En 20IO-20I I, grâce à la générosité de Denis Fisette, est paru un numéro qui contient mes trois conférences Hugues Leblanc (Philosophiques, 38, 2). Ces conférences font partie de Wittgenstein et la philosophie austro-allemande (désormais "WPAA»), dont Philosophiques publie maintenant mon précis ainsi que quatre critiques, rassemblées à l'initiative de Jimmy Plourde, par Plourde lui-même, Guillaume Fréchette, Patrice Philie, Denis Seron, et me donne la possibilité d'y répondre.

Que Philosophiques, Denis Fisette ainsi que mes quatre critiques soient vivement remerciés!

WPAA est le fruit d'un certain étonnement. L'énorme industrie exégétique consacrée aux réflexions du dernier Wittgenstein au sujet de l'esprit, du langage et des couleurs semble ignorer que les sujets, thèses et distinctions abordés par Wittgenstein étaient en grande partie discutés pour la première fois par les héritiers de Franz Brentano - en particulier Husserl, Pfänder, Meinong, Scheler, Geiger, Reinach, Katz, Köhler, Bühler et Ortega y Gasset - et non pas, par exemple, par les autres pères de la philosophie analytique. Entre la fin du dix-neuvième siècle et les années trente, l'un ou l'autre des héritiers de Brentano au moins propose une philosophie des règles linguistiques, de la variété des emplois des mots, du fait de vouloir dire quelque chose avec une expression, des ressemblances de famille, des pouvoirs, du voir comme, des attitudes, du rapport entre le brun, la transparence et le solide des couleurs et de la compréhension d'une culture, Weltanschauung ou forme de vie. Il en va de même des distinctions suivantes:

- concepts formels par rapport à concepts matériels

- relations externes par rapport aux relations internes

- essence par rapport à modalité

- sens par rapport à non-sens

1. 2012, Paris, Vrin

PHILOSOPHIQUES 42/2 - Automne 2015, p. 359-365 
- raisons par rapport à motifs par rapport à justification par rapport à objets par rapport à causes

- critères par rapport à symptômes

- objets privés par rapport à objets publics par rapport à non-objets

- bouger son bras par rapport au mouvement d'un bras

- expressions indexicales par rapport aux noms propres

- certitudes primitives par rapport à certitudes critiques

Ce fait, s'il en est un, présente un certain intérêt pour celui qui veut comprendre Wittgenstein et la philosophie du vingtième siècle. À la communauté de sujets, questions, distinctions et problèmes mentionnée s'ajoute le fait que tant les héritiers de Brentano que Wittgenstein se réclament de façon bruyante de la méthode de la description. Mais ce que Wittgenstein fait des distinctions et sujets mentionnés n'est pas du tout ce qu'en font les héritiers de Brentano. Les descriptions données par Wittgenstein et les héritiers de Brentano sont néanmoins comparables quant à la question de leur portée. Si Wittgenstein est persuadé que ses descriptions ne font que révéler la complexité et la variété des phénomènes étudiés, les psychologues descriptifs, les phénoménologues et les psychologues de la Gestalt sont persuadés que leurs descriptions et analyses révèlent de l'ordre et du système.

D'où les trois questions auxquelles répond WPAA. Les distinctions, thèses et sujets abordés par le dernier Wittgenstein furent-ils vraiment en grande partie anticipés par ses contemporains et prédécesseurs autrichiens et allemands? Jusqu'où va la similarité entre leurs méthodes descriptives? Les descriptions données par Wittgenstein montrent-elles que les buts théoriques et systématiques poursuivis par ses contemporains et prédécesseurs étaient vains? Si les deux premières questions relèvent de l'histoire de la philosophie et de l'exégèse, la dernière question est une question philosophique.

Le premier chapitre - «Descriptions, différences et découvertes »explore, d'un côté, les rapports entre la priorité de la description, et, de l'autre côté, l'explication, les hypothèses, les différences, les découvertes, les trivialités, la rhétorique intuitionniste, l'idée d'une übersichtliche Darstellung, ainsi que les images trompeuses en philosophie, selon Wittgenstein et ses prédécesseurs. Les nombreuses similarités notées contrastent de façon dramatique avec deux conceptions très différentes de l'objet de la description, dans les deux sens de cette expression. D’abord, les héritiers de Brentano poursuivent la description et l'analyse dans le but d'arriver à des vérités philosophiques et systématiques. Wittgenstein poursuit la description non pas dans un but théorique, mais pour nous guérir de toute tentation théorique et philosophique. Ensuite, si les phénoménologues et Wittgenstein cherchent l'essence du vouloir dire ou des émotions, Wittgenstein, à la différence des phénoménologues, veut nous montrer que l'essence se trouve dans la façon dont les mots sont employés ou sont à employer, 
dans la grammaire. Là où les héritiers de Brentano croient être en train de décrire la complexité psychologique et mentale, Wittgenstein croit être en train de décrire les emplois des mots.

Le chapitre II — «De l'Esprit, de l'âme et des mondes» — introduit la distinction entre les catégories d'objets publics, d'objets privés et de «nonobjets» tracée par Scheler dans son Formalisme (I9I3-I9I6) et ailleurs. Il s'agit d'une nouveauté dans la tradition remontant à Brentano: il n'y a pas, selon Scheler, d'états ou de phénomènes psychologiques qui soient épistémologiquement privés. Un «acte mental», par contre, tel que vouloir dire quelque chose, ne serait ni un objet privé ni un objet public, mais quelque chose qui peut être compris et connu sans devenir un objet connu, dans une forme de "knowledge without observation» ou "conscience non thétique». La distinction de Scheler est comparée à la nature du vouloir dire et du penser selon le Tractatus de Wittgenstein, ainsi qu'à une distinction analogue tracée par Wittgenstein bien après le Tractatus.

Le chapitre III — «Éprouver vs Vouloir Dire, Vouloir» — étudie ce qui semble être un objet public et psychologique tant selon Scheler que selon Wittgenstein, à savoir les émotions, telles que la tristesse au sujet de quelque chose, et deux exemples de la catégorie de non-objets, vouloir dire (Meinen, mean) et vouloir, selon Reinach, Scheler et Wittgenstein. Selon Scheler et Wittgenstein, la tristesse de Sam est un état qui peut être perçu par d'autres personnes grâce à la nature de la relation d'expression. Le fait de vouloir dire quelque chose est le sujet de nombreuses réflexions de Husserl - qui semble avoir introduit ce phénomène dans la philosophie moderne -, et de Reinach, Scheler et Wittgenstein. Le verbe mental qui intéresse Wittgenstein plus que n'importe quel autre est bien «Meinen» (et «mean»). Selon Reinach, puis Wittgenstein, vouloir dire n'est pas un processus mental. Les deux penseurs tirent plusieurs conséquences semblables de ce fait. Mais la description du vouloir dire que donnent Reinach et Scheler met ce phénomène au centre d'une philosophie systématique des «actes» mentaux.

Les chapitres IV-VI sont consacrés au sens (Sinn) et à la signification (Bedeutung).

Le chapitre IV — «Sens, non-sens, et modalités » — étudie la géographie du sens et de la proposition selon le Tractatus, l'arrière-plan de la description que donnera Wittgenstein plus tard de la signification. Elle est comparée à l'autre géographie autrichienne du sens, celle donnée en I90 I par Husserl dans ses Recherches logiques. Le rapport très complexe entre ces deux philosophies pionnières du non-sens est étudié surtout relativement à un certain nombre de thèses ontologiques présentées par les deux penseurs. Elles sont de trois types. Elles concernent les possibilités et impossibilités essentielles sur le plan de ce qui est et n'est pas doué de sens, sur le plan des objets, états de choses et situations, et elles concernent les possibilités et impossibilités essentielles qui relient ces deux plans. Husserl, par exemple, affirme, et Wittgenstein nie qu'un état de choses ou une situation 
puisse être nommé. Selon Husserl, la possibilité de passer de la description d'un état de choses à une prédication qui contient un nom de cet état de choses est l'une des nombreuses possibilités essentielles de montée ( «ascent») sémantique garantie par sa théorie des modifications ou transformations sémantiques et de l'essence des propositions. Si la montée n'est possible qu'à la condition qu'il y ait des propositions abstraites, un philosophe qui rejette de telles propositions devrait rejeter la possibilité de la montée. Wittgenstein rejette et les propositions abstraites et plusieurs types de montée.

Le chapitre V - «Significations» — analyse les différentes significations de "Bedeutung» dans la philosophie austro-allemande, examine le rapport intime que voient d'abord Bühler puis Wittgenstein entre la fonction linguistique de représenter ( darstellen») un état de choses à l'aide d'une phrase ou une proposition, et la description, les rapports entre les significations et les règles selon Ahlman et le dernier Wittgenstein, et la signification secondaire selon Meinong et Wittgenstein.

Le chapitre VI - «Des mots et des dalles» — confronte la théorie du langage de Bühler, exposée de la façon la plus complète dans sa Théorie du langage de 1934, et les remarques sur le langage au début des Recherches philosophiques de Wittgenstein. Selon Bühler, les langues naturelles ont une essence ou nature qu'il prétend cerner à l'aide d'une taxinomie remarquable des fonctions linguistiques - celle déjà mentionnée de représenter (darstellen), celle d'exprimer ou manifester les états mentaux et celle de guider ou influencer un interlocuteur - ainsi que des différents types de signes et de contextes, linguistiques et non linguistiques. Wittgenstein, par contre, nie que le langage possède une nature ou essence. Le résultat de la confrontation est que les différents types d'emplois de mots décrits par Wittgenstein s'avèrent être des illustrations bien choisies de la taxinomie proposée par Bühler. Bühler et Wittgenstein attachent, en particulier, une grande importance au phénomène de guidage (Steuerung) ou d'influence dans toute sa variété. Mais si selon Bühler la fonction de représenter les états de choses est une fonction irréductible, selon Wittgenstein la représentation est loin d'être un phénomène irréductible. Les considérations avancées par Wittgenstein en faveur de cette conclusion avaient été considérées par Bühler qui, à juste titre, les rejette. La théorie du langage de Bühler est le dernier avatar d'un certain courant philosophique, la théorie empirico-téléologique du langage, qui remonte aux travaux de Marty et de Brentano. La théorie des règles linguistiques d'Ahlman, et en particulier la thèse selon laquelle la signification est déterminée par de telles règles, théorie présentée dans le chapitre $\mathrm{V}$, appartient elle aussi à cette tradition. Mais Bühler, à la différence d'Ahlman, a fait siennes les analyses de la référence démonstrative sur la base de la perception développées par Husserl, qui l'amènent à parler, comme Wittgenstein, des composantes non verbales du langage, ainsi que la conception husserlienne du vouloir dire. 
Le chapitre VII — «Couleurs» — examine les remarques de Wittgenstein au sujet des couleurs telles qu'elles sont représentées par le solide des couleurs et les couleurs qui jouent un rôle dans la perception ordinaire. Les premières discussions wittgensteiniennes de l'espace des couleurs, de la différence entre le carré et le cercle des couleurs, nous montrent à quel point la différence entre les relations internes et les relations externes est toujours centrale dans sa pensée, et pourquoi il a été obligé de réviser ses idées dans le Tractatus au sujet des relations internes entre les couleurs, telles que l'incompatibilité. Or les distinctions, thèses et problèmes discutés par Wittgenstein sont en grande partie ceux discutés par Meinong en 1903, dans son analyse de l'octaèdre de son élève Höfler. L'essentialisme de Meinong au sujet de l'espace des couleurs - et d'autres espaces encore — n'est certainement pas partagé par Wittgenstein, mais sur le plan de la description et de l'analyse les deux penseurs autrichiens sont souvent d'accord.

Les remarques ultérieures de Wittgenstein au sujet des couleurs non réduites de tous les jours représentent l'une de ses tentatives les plus développées pour montrer la variété sans système d'un domaine de phénomènes. Mais rien dans les descriptions de Wittgenstein ne permet d'infirmer l'analyse systématique des couleurs donnée par Katz, un élève de Husserl, et les compléments apportés par Bühler. La complexité des rapports entre la couleur et la lumière est très grande, estiment Katz et Bühler, mais elle n'est pas désordonnée. Presque toutes les descriptions que Wittgenstein donne des couleurs trouvent leur place dans le système élaboré par Katz en I9 I I et par la suite.

Le chapitre VIII — «Certitudes» — étudie les relations entre les quatre descriptions de la certitude primitive données pendant la première moitié du vingtième siècle - par Husserl, Scheler, Ortega et Wittgenstein. Selon les quatre penseurs, il y a des croyances ou certitudes qui sont à la fois sans justification et très importantes dans la vie de tous les jours. L'analyse la plus poussée est celle d'Ortega, qui étudie d'ailleurs le plus grand nombre de types de certitude primitive, par exemple les certitudes perceptuelles, la certitude en action et les certitudes normatives. Les rôles des certitudes primitives identifiées par Wittgenstein sont presque tous notés par ses trois prédécesseurs. Mais la philosophie systématique des "croyances de base" que donne Ortega diffère sur plusieurs points importants de l'analyse de la «certitude naïve» que donne Husserl.

$\mathrm{La}$ "Conclusion» de WPAA souligne que le livre n'avance aucune thèse concernant les supposées «sources» de Wittgenstein. Ceux qui souffrent de ce que Koyré a appelé le "virus du précurseur " seront sans doute intéressés par les témoignages des philosophes les mieux qualifiés pour parler de telles «sources».

D'abord Bergmann et Waismann, selon un compte rendu de Bergmann de 1939 adressé à Neurath. Bergmann distingue la "période classique » du Cercle de Vienne de sa période ultérieure. Pendant la première phase, deux 
phénoménologues, Kaufmann et Neumann, participaient régulièrement aux discussions du Cercle:

[W] hen they took the floor, during the classical period, Schlick occasionally showed a certain impatience and sometimes even interrupted the discussion outright. It is all the more interesting that, as the Wittgensteinian ideas emerged into the foreground, the phenomenological group gained ground as they claimed to recognize many of the ideas now advanced as old teaching (altes Lehrgut) from their own school. To be sure, this was rejected by Schlick and Waismann, although Waismann at that time in private already recommended reading Husserl, in whom much that is essential was said to be anticipated (vorgefüblt). It was from this side, moreover, that criticism set in. In the Circle, Hahn once asked Waismann directly how he distinguished himself any more from a phenomenologist ${ }^{2}$.

La réponse de Waismann, s'il y en a eu une, n'a malheureusement pas été préservée.

Ensuite Ryle, l'une des deux personnes qui, selon Wittgenstein, l'avaient (à un moment donné) compris:

It was, I think, Meinong and, hand in hand with him, the young Russell who brought back into philosophy the many-strandedness of Aristotle. Meinong's work did have its scholastic dessication, but it had as well its Aristotelian multifariousness.I believe, but do not argue, that this multifariousness, without this dessication, was one confluent into Wittgenstein's Philosophical Investigations ${ }^{3}$.

Enfin, Findlay, un élève de Wittgenstein et l'auteur d'un livre sur Meinong, estime que Brentano, Husserl et Meinong avaient

a great influence on Russell and Moore, and many of their opinions filtered through to Wittgenstein from these and other Cambridge sources and became targets of criticism at many points in his work ${ }^{4}$.

Ryle et Findlay connaissaient très bien la psychologie descriptive et en particulier les philosophies de Meinong et de Husserl. Bergmann ne connaissait pas encore ces deux philosophies, mais est devenu par la suite l'un des commentateurs les plus importants de Husserl et de Meinong.

Les témoignages de Bergmann, Waismann, Findlay et Ryle ne semblent pas avoir eu le moindre effet sur l'industrie exégétique consacrée à Wittgenstein. D'où l'étonnement à l'origine de WPAA. Un étonnement non moins grand semble approprié lorsque l'on constate l'extraordinaire manque d'intérêt manifesté par l'industrie exégétique phénoménologique pour la plupart des développements philosophiques inspirés de Brentano. Cette double

2. Bergmann I993, p. 200 (I988, p. I7); je souligne - KM.

3. Ryle I972, p. I4.

4. Findlay 1984 , p. 22 ; je souligne - KM. 
cécité au sujet de l'histoire de la philosophie durant le vingtième siècle n'a pas été, me semble-t-il, bonne pour la philosophie elle-même.

\section{Bibliographie}

Bergmann, G. "Erinnerungen an den Wiener Kreis. Brief an Otto Neurath", with an introduction by Reinhardt Grossmann, in Vertriebene Vernunft, II. Emigration und Exil österreichischer Wissenschaftler, F. Stadler (ed.), Vienna \& Munich: Jugend und Volk, I988, pp. I7I-I80. (English translation: "Memories of the Vienna Circle. Letter to Otto Neurath (1938) ", in Scientific Philosophy: Origins and Developments, F. Stadler (ed.), Vienna Circle Institute Yearbook, I, 1993, Kluwer, pp. 193-208).

Findlay, J. N. Wittgenstein: A Critique, London: Routledge and Kegan Paul, I984. 